\title{
p-Adic and Adelic Cosmology: $p$-Adic Origin of Dark Energy and Dark Matter
}

\author{
Branko Dragovich \\ Institute of Physics, P.O.Box 57, 11001 Belgrade, SERBIA AND MONTENEGRO \\ email: dragovich@phy.bg.ac.yu
}

\begin{abstract}
A brief review of $p$-adic and adelic cosmology is presented. In particular, $p$-adic and adelic aspects of gravity, classical cosmology, quantum mechanics, quantum cosmology and the wave function of the universe are considered.

$p$-Adic worlds made of $p$-adic matters, which are different from real world of ordinary matter, are introduced. Real world and $p$-adic worlds make the universe as a whole. $p$-Adic origin of the dark energy and dark matter are proposed and discussed.
\end{abstract}

\section{INTRODUCTION}

Cosmology in its own right is a science devoted to the universe as a whole. It is based on the cosmological observational data and fundamental physical theories (especially: general theory of relativity, quantum theory and theory of elementary particles). So far many significant results have been obtained and the Standard Cosmological Model is established (see, e.g. [1]). According to this model at the very beginning the universe was very small, dense, hot and started to expand. This initial period of evolution should be described by quantum cosmology. According to general theory of relativity the universe is a (pseudo)Riemannian space, which is presently flat. From observational data follows that the universe has permanently expanded during all its history which is about 14 billion years. Since 1998, there are a lot of evidence that the universe is now in the stage of an accelerated expansion which began a few billion years ago (for a review, see $[2,3])$. To explain this acceleration many models have been proposed but no one of them was generally accepted. A very natural and the most attractive is the approach with dark energy, which is a matter with a negative pressure and uniformly distributed in the space. About $70 \%$ of all energy content of the universe is related to the dark energy, while about $26 \%$ belongs to the dark matter and only $4 \%$ is made of baryons (protons and neutrons). However the nature of the dark energy is a big mystery, which presents one of the greatest problems and challenges of contemporary cosmology and theory of elementary particles.

It is well known that results of observational measurements, as well as of the experimental ones, are elements of the field $\mathbb{Q}$ of rational numbers. However theoretical models are constructed not over $\mathbb{Q}$ but traditionally over $\mathbb{R}$ (field of real numbers) or $\mathbb{C}$ (field of 
complex numbers), where $\mathbb{R}$ is completion of $\mathbb{Q}$ with respect to distance induced by the ordinary absolute value $|\cdot|_{\infty}$ and $\mathbb{C}$ is algebraic extension of $\mathbb{R}$. Thus it is worth noting that mathematical modeling of physical systems requires not only algebraic structure of $\mathbb{Q}$ but also its geometric properties, which are related to the possible norms on $\mathbb{Q}$. Besides $|\cdot|_{\infty}$ there are also infinitely many $p$-adic norms $|\cdot|_{p}$, i.e. there is one nontrivial and inequivalent norm for each prime number $p[4,5]$.

Measurements represent comparison of a given quantity with respect to a fixed quantity of the same nature taken to be the unit one. We live in the world where our measurements are inherently connected with the (decimal) expansions of the real numbers. Namely, in the process of measurement one determines a finite number of digits in the decimal expansion. This number of digits can be enlarged using more precise tools, while all other digits remain hidden within measuring errors. According to the classical mechanics there are no in principle physical restrictions to measure all quantities of a system with arbitrary accuracy. However in quantum mechanics there is restriction as a consequence of the well known uncertainty relation $\Delta x \Delta k \geq \hbar / 2$. When quantum mechanics is combined with gravity then there obtains strong restriction on measurement of very small distances in the form

$$
\Delta x \geq \ell_{0}=\sqrt{\frac{G \hbar}{c^{3}}} \sim 10^{-33} \mathrm{~cm},
$$

where $\ell_{0}$ is the Planck length. It follows that there is quite firm restriction to measure spatial distances with arbitrary accuracy. In other words, it is not possible to determine all digits related to positions in the space. Thus measurements of physical quantities up to the Planck scale give rational numbers with geometrical properties which can be described by the usual absolute value. Hence it has been natural to use real numbers and complex numbers to describe physical phenomena in the explored domain of the universe. It is not strange that just real analysis is used to describe these real data. However for a more profound physical theory there is a sense to employ also $p$-adic numbers $[4,6]$, which are completions of $\mathbb{Q}$ with respect to the $p$-adic norms.

If we cannot get as a result of direct measurement a rational number with $p$-adic norm properties, it does not mean that there is not any content of the universe which natural description is just by $p$-adic numbers. Suppose that such $p$-adic systems exist. Then real number, as a result of measurement, is a real measure of interaction between $p$ adic and real system, to which belong measuring instruments. In such case we have to use analysis with $p$-adic valued functions of $p$-adic argument to describe $p$-adic system itself and also to use analysis with real (complex) valued functions of $p$-adic argument to describe $p$-adic system from the real point of view. This is slightly similar to the employment of complex functions in quantum mechanics, where wave function $\psi(x) \in \mathbb{C}$ contains complete information on quantum system, but is not measurable quantity, while $|\psi(x)|^{2} \in \mathbb{R}$ is related to the probability distribution which can be measured. Let us use terms real and $p$-adic to denote those aspects of the universe which can be naturally described by real and $p$-adic numbers, respectively. We conjecture here that the visible and dark sides of the universe are real and $p$-adic ones, respectively.

p-Adic strings were introduced in 1987 [7] and a nice adelic formula was obtained [8]. An effective theory with real numbers of $p$-adic strings was constructed $[9,10]$ and 
shown its importance in the context of the tachyon condensation [11]. Application of $p$ adic numbers in construction of various models for the first five years was presented in [12] and [6]. Current activity is reflected in the proceedings of the international conferences on $p$-adic mathematical physics $[13,14]$.

In Section 2 we give an introductory review of adeles as well as of $p$-adic and adelic analogs of classical cosmology. Sec. 3 is devoted to $p$-adic and adelic quantum mechanics and their employment in quantum cosmology. $p$-Adic matter and its cosmological aspects are considered in Sec. 4.

\section{2. $p$-ADIC AND ADELIC CLASSICAL COSMOLOGY}

Adelic classical cosmology is a generalization of the ordinary one (over real numbers) in such way that it employs all (real and $p$-adic) completions of the set of rational numbers $\mathbb{Q}$. It uses analysis based on adelic valued functions of adelic valued arguments.

Let us remind some basic properties of adeles and adelic valued functions. To consider real and $p$-adic numbers simultaneously and on equal footing one uses concept of adeles. An adele $x$ (see, e.g. [5]) is an infinite sequence

$$
x=\left(x_{\infty}, x_{2}, \cdots, x_{p}, \cdots\right), \quad x_{\infty} \in \mathbb{R}, x_{p} \in \mathbb{Q}_{p}
$$

with the restriction that for all but a finite set $\mathscr{P}$ of primes $p$ must be $x_{p} \in \mathbb{Z}_{p}$, where $\mathbb{Z}_{p}=\left\{\left.y \in \mathbb{Q}_{p}|| y\right|_{p} \leq 1\right\}$ is the ring of $p$-adic integers. Componentwise addition and multiplication endow the ring structure to the set of all adeles $\mathbb{A}$, which is the union of direct products in the following form:

$$
\mathbb{A}=\bigcup_{\mathscr{P}} \mathbb{A}(\mathscr{P}), \quad \mathbb{A}(\mathscr{P})=\mathbb{R} \times \prod_{p \in \mathscr{P}} \mathbb{Q}_{p} \times \prod_{p \notin \mathscr{P}} \mathbb{Z}_{p}
$$

A multiplicative group of ideles $\mathbb{I}$ is a subset of $\mathbb{A}$ with elements $x=\left(x_{\infty}, x_{2}, \cdots, x_{p}, \cdots\right)$, where $x_{\infty} \in \mathbb{R}^{*}=\mathbb{R} \backslash\{0\}$ and $x_{p} \in \mathbb{Q}_{p}^{*}=\mathbb{Q}_{p} \backslash\{0\}$ with the restriction that for all but a finite set $\mathscr{P}$ one has $x_{p} \in \mathbb{U}_{p}$, where $\mathbb{U}_{p}=\left\{\left.y \in \mathbb{Q}_{p}|| y\right|_{p}=1\right\}$ is the multiplicative group of $p$-adic units. Thus the whole set of ideles is

$$
\mathbb{I}=\bigcup_{\mathscr{P}} \mathbb{I}(\mathscr{P}), \quad \mathbb{I}(\mathscr{P})=\mathbb{R}^{*} \times \prod_{p \in \mathscr{P}} \mathbb{Q}_{p}^{*} \times \prod_{p \notin \mathscr{P}} \mathbb{U}_{p}
$$

A principal adele (idele) is a sequence $(x, x, \cdots, x, \cdots) \in \mathbb{A}$, where $x \in \mathbb{Q} \quad\left(x \in \mathbb{Q}^{*}=\right.$ $\mathbb{Q} \backslash\{0\}) . \mathbb{Q}$ and $\mathbb{Q}^{*}$ are naturally embedded in $\mathbb{A}$ and $\mathbb{I}$, respectively.

Let us define an ordering on the set $\mathbb{P}$, which consists of all finite sets $\mathscr{P}_{i}$ of primes $p$, by $\mathscr{P}_{1} \prec \mathscr{P}_{2}$ if $\mathscr{P}_{1} \subset \mathscr{P}_{2}$. It is evident that $\mathbb{A}\left(\mathscr{P}_{1}\right) \subset \mathbb{A}\left(\mathscr{P}_{2}\right)$ when $\mathscr{P}_{1} \prec \mathscr{P}_{2}$. Spaces $\mathbb{A}(\mathscr{P})$ have natural Tikhonov topology and adelic topology in $\mathbb{A}$ is introduced by inductive limit: $\mathbb{A}=\operatorname{limind} \mathscr{P} \in \mathbb{P} A(\mathscr{P})$. A basis of adelic topology is a collection of open sets of the form $W(\mathscr{P})=\mathbb{V}_{\infty} \times \prod_{p \in \mathscr{P}} \mathbb{V}_{p} \times \prod_{p \notin \mathscr{P}} \mathbb{Z}_{p}$, where $\mathbb{V}_{\infty}$ and $\mathbb{V}_{p}$ are open 
sets in $\mathbb{R}$ and $\mathbb{Q}_{p}$, respectively. Note that adelic topology is finer than the corresponding Tikhonov topology. A sequence of adeles $a^{(n)} \in \mathbb{A}$ converges to an adele $a \in \mathbb{A}$ if $(i)$ it converges to $a$ componentwise and (ii) if there exist a positive integer $N$ and a set $\mathscr{P}$ such that $a^{(n)}, a \in \mathbb{A}(\mathscr{P})$ when $n \geq N$. In the analogous way, these assertions hold also for idelic spaces $\mathbb{I}(\mathscr{P})$ and $\mathbb{I}$.

Adelic valued functions of adelic arguments are maps $F_{\mathbb{A}}: U_{\mathbb{A}} \rightarrow V_{\mathbb{A}}$, where $U_{\mathbb{A}} \subset \mathbb{A}^{n}$, $V_{\mathbb{A}} \subset \mathbb{A}^{m}$ and have the form

$$
F_{\mathbb{A}}(x)=\left(f_{\infty}\left(x_{\infty}\right), f_{2}\left(x_{2}\right), \cdots, f_{p}\left(x_{p}\right), \cdots\right), \quad f_{\infty} \in \mathbb{R}^{m}, f_{p} \in \mathbb{Q}_{p}^{m},
$$

where for all but $p \in \mathscr{P}$ one has to satisfy $\left|f_{p}\left(x_{p}\right)\right|_{p} \leq 1$.

When $F_{\mathbb{A}}(x)$ is related to the same physical system it is natural to expect that $v$-adic $(v=\infty, 2, \cdots, p, \cdots)$ functions $f_{v}\left(x_{v}\right)$ have the same form of dependence on $x_{v}$. As an illustrative example one can take adelic valued exponential function

$$
\exp _{\mathbb{A}} x=\left(\exp _{\infty} x_{\infty}, \exp _{2} x_{2}, \cdots, \exp _{p} x_{p}, \cdots\right)
$$

where $v$-adic exponential functions are defined by the usual power series expansion

$$
\exp _{v} x_{v}=\sum_{n=0}^{\infty} \frac{x_{v}^{n}}{n !}, \quad\left|x_{p}\right|_{p} \leq|2 p|_{p}, \quad\left|\exp _{p} x_{p}\right|_{p}=1
$$

Similar situation is for functions $\sin _{\mathbb{A}} x, \cos _{\mathbb{A}} x, \sinh _{\mathbb{A}} x, \cosh _{\mathbb{A}} x$ and many other functions given by power series expansions with rational coefficients.

The Einstein gravitational field equations

$$
R_{\mu v}-\frac{1}{2} R g_{\mu v}=\kappa T_{\mu v}-\Lambda g_{\mu v}
$$

can be also considered as $p$-adic ones if we take constants $\kappa=\frac{8 \pi G}{c^{4}}$ and $\Lambda$ to be rational numbers. By this way the Einstein equations (8) become number field invariant and therefore more fundamental. A successful systematic study of $p$-adic gravity, especially $p$-adic differential geometry and gravitational field equations, started in 1991 [15].

For the sequel it is useful to introduce $\pi G=\bar{G}$ and the new Planck quantities

$$
L_{0}=\sqrt{\frac{h \bar{G}}{c^{3}}}=\sqrt{2} \pi \ell_{0}, \quad T_{0}=\frac{L_{0}}{c}=\sqrt{2} \pi t_{0}, \quad M_{0}=\sqrt{\frac{h c}{\bar{G}}}=\sqrt{2} m_{0},
$$

where $\ell_{0}, t_{0}$ and $m_{0}$ are usual Planck length, time and mass, respectively. Let us take for the natural system of units these $L_{0}, T_{0}$ and $M_{0}$ instead of the standard $\ell_{0}, t_{0}$ and $m_{0}$. Then $\bar{G}, c$ and $h$ are rational numbers with unit values.

Constructing cosmological models it is useful to maximally exploit symmetrical properties of the universe. As a consequence one obtains dynamical system called minisuperspace cosmological model, which contains finite number degrees of freedom. Minisuperspace model may be regarded as a classical system given by Hamiltonian $H\left(q^{i}, k^{i}, t\right)$ 
or Lagrangian $L\left(q^{i}, \dot{q}^{i}, t\right)$, where $i=1,2, \cdots, n$. The corresponding adelic Hamiltonian is

$$
H_{\mathbb{A}}\left(q^{i}, k^{i}, t\right)=\left(H_{\infty}\left(q_{\infty}^{i}, k_{\infty}^{i}, t_{\infty}\right), H_{2}\left(q_{2}^{i}, k_{2}^{i}, t_{2}\right), \cdots, H_{p}\left(q_{p}^{i}, k_{p}^{i}, t_{p}\right), \cdots\right),
$$

where $H_{\infty} \in \mathbb{R}$ and $H_{p} \in \mathbb{Q}_{p}$ with restriction that $H_{p} \in \mathbb{Z}_{p}$ for all but $p \in \mathscr{P}$. Analogously one defines an adelic Lagrangian. $v$-Adic action of the minisuperspace model is

$$
S_{v}[q]=\int_{t^{\prime}}^{t^{\prime \prime}} d t N\left[\frac{1}{2 N^{2}} f_{\alpha \beta}(q) \dot{q}^{\alpha} \dot{q}^{\beta}-U(q)\right]
$$

where $f_{\alpha \beta}$ is a metric on minisuperspace of some gravitational and matter field variables. The de Sitter minisuperspace cosmological model is a simple nontrivial, exactly solvable and instructive cosmological model. It is given by the Einstein-Hilbert action with cosmological constant $\Lambda(c=1)$,

$$
S\left[g_{\mu \nu}\right]=\frac{1}{16 \pi G}\left[\int_{M} d^{4} x \sqrt{-g}(R-2 \Lambda)+2 \int_{\partial M} d^{3} x \sqrt{h} K\right]
$$

and the Friedmann-Robertson-Walker (FRW) metric

$$
d s^{2}=-N^{2} d t^{2}+a^{2}(t) d \Omega_{3}^{2}
$$

where $N(t)$ is the lapse function and $d \Omega_{3}^{2}$ is the metric on the unit three-sphere. More complex models contain also additional action with matter fields. To simplify formalism and get quadratic Lagrangian one can take the Robertson-Walker metric in the form [17]

$$
d s^{2}=-\frac{N^{2}}{q(t)} d t^{2}+q(t) d \Omega_{3}^{2} .
$$

Using metric (14) in (12), one obtains

$$
S[q]=\frac{1}{2} \int_{t^{\prime}}^{t^{\prime \prime}} d t N\left(-\frac{\dot{q}^{2}}{4 N^{2}}-\lambda q+1\right)
$$

where $\lambda=\Lambda / 3$. Choosing $N=1$, the equation of motion is

$$
\ddot{q}=2 \lambda \text {. }
$$

Solution of the equation (16) which satisfies conditions $q^{\prime \prime}=q(T)$ and $q^{\prime}=q(0)$ is

$$
q(t)=\lambda t^{2}+\left(\frac{q^{\prime \prime}-q^{\prime}}{T}-\lambda T\right) t+q^{\prime}
$$

Note that equations (16) and (17) are the same as for a particle with constant acceleration $a=2 \lambda$. The corresponding classical action (see, e.g. [16] and references therein) is

$$
\bar{S}[q]=\frac{\lambda^{2} T^{3}}{24}-\left[\lambda\left(q^{\prime \prime}+q^{\prime}\right)-2\right] \frac{T}{4}-\frac{\left(q^{\prime \prime}-q^{\prime}\right)^{2}}{8 T} .
$$


The above consideration of the de Sitter model is performed for the real case but the expressions from (15) to (18) can be also regarded as $p$-adic valued.

Since parameter $\lambda=\frac{\Lambda}{3}$ has rational values we can write adelic Lagrangian in the form

$$
L_{\mathbb{A}}(q, \dot{q})=\left(L_{\infty}\left(q_{\infty}, \dot{q}_{\infty}\right), L_{2}\left(q_{2}, \dot{q}_{2}\right), \cdots, L_{p}\left(q_{p}, \dot{q}_{p}\right), \cdots\right)
$$

where $(N=1)$

$$
L_{v}\left(q_{v}, \dot{q}_{v}\right)=\frac{1}{2}\left(-\frac{\dot{q}_{v}^{2}}{4}-\lambda q_{v}+1\right) .
$$

It is evident that $\left|L_{p}\left(q_{p}, \dot{q}_{p}\right)\right|_{p} \leq 1$ when $\left|q_{p}\right|_{p} \leq 1,\left|\dot{q}_{p}\right|_{p} \leq 1,|\lambda|_{p} \leq 1$ and $p \neq 2$. Infinite sequence of actions

$$
\bar{S}_{\mathbb{A}}[q]=\left(\bar{S}_{\infty}\left[q_{\infty}\right], \bar{S}_{2}\left[q_{2}\right], \cdots, \bar{S}_{p}\left[q_{p}\right], \cdots\right),
$$

where $\bar{S}_{v}\left[q_{v}\right],(v=\infty, 2, \cdots, p, \cdots)$ are given by (18), becomes adelic if $T$ is principal idele and $q^{\prime \prime}, q^{\prime}$ are adeles.

For some other $p$-adic and adelic quadratic classical cosmological models see [16].

\section{3. $p$-ADIC AND ADELIC QUANTUM COSMOLOGY}

$p$-Adic and adelic quantum cosmology $[15,18,19,16]$ is an appropriate application of $p$-adic [20] and adelic quantum mechanics [21, 22, 23] to the universe as a whole at its very early stage of evolution. Let us now first recall the basic properties of $p$-adic and adelic quantum mechanics.

\section{1. $p$-Adic and Adelic Quantum Mechanics}

It is remarkable that ordinary quantum mechanics on a real space can be generalized to quantum mechanics on $p$-adic spaces for any prime number $p$. There are two main approaches: with complex-valued [20] and $p$-adic valued [24] elements of the Hilbert space on $\mathbb{Q}_{p}^{n}$. p-Adic quantum mechanics with complex-valued wave functions is more suitable for connection with ordinary quantum mechanics, and in the sequel we will briefly review only this kind of $p$-adic quantum mechanics.

When wave functions are complex-valued and arguments are $p$-adic valued, one cannot construct a direct analog of the Schrödinger equation with a $p$-adic version of Heisenberg algebra. According to the Weyl quantization, canonical noncommutativity in $p$-adic case can be introduced by operators $(h=1)$

$$
\hat{Q}_{p}(\alpha) \psi_{p}(x)=\chi_{p}(-\alpha x) \psi_{p}(x), \quad \hat{K}_{p}(\beta) \psi_{p}(x)=\psi_{p}(x+\beta)
$$

which satisfy

$$
\hat{Q}_{p}(\alpha) \hat{K}_{p}(\beta)=\chi_{p}(\alpha \beta) \hat{K}_{p}(\beta) \hat{Q}_{p}(\alpha)
$$


where $\chi_{p}(u)=\exp \left(2 \pi i\{u\}_{p}\right)$ is additive character on the field $\mathbb{Q}_{p}$ and $\{u\}_{p}$ is the fractional part of $u \in \mathbb{Q}_{p}$.

Let $\hat{x}$ and $\hat{k}$ be operators of position $x$ and momentum $k$, respectively. Let us define operators $\chi_{v}(\alpha \hat{x})$ and $\chi_{v}(\beta \hat{k})$ by formulas

$$
\chi_{v}(\alpha \hat{x}) \chi_{v}(a x)=\chi_{v}(\alpha x) \chi_{v}(a x), \quad \chi_{v}(\beta \hat{k}) \chi_{v}(b k)=\chi_{v}(\beta k) \chi_{v}(b k),
$$

where index $v$ denotes real and any $p$-adic case, and $\chi_{\infty}(u)=\exp (-2 \pi i u)$. These operators also act on a function $\psi_{v}(x)$, which has the Fourier transform $\tilde{\psi}(k)$, in the following way:

$$
\begin{gathered}
\chi_{v}(-\alpha \hat{x}) \psi_{v}(x)=\chi_{v}(-\alpha \hat{x}) \int \chi_{v}(-k x) \tilde{\psi}(k) d^{n} k=\chi_{v}(-\alpha x) \psi_{v}(x), \\
\chi_{v}(-\beta \hat{k}) \psi_{v}(x)=\int \chi_{v}(-\beta k) \chi_{v}(-k x) \tilde{\psi}(k) d^{n} k=\psi_{v}(x+\beta),
\end{gathered}
$$

where integration in $p$-adic case is with respect to the Haar measure $d k$ with the properties: $d(k+a)=d k, d(a k)=|a|_{p} d k$ and $\int_{|k|_{p} \leq 1} d k=1$. Comparing (22) with (25) and (26) we conclude that $\hat{Q}_{p}(\alpha)=\chi_{p}(-\alpha \hat{x}), \hat{K}_{p}(\beta)=\chi_{p}(-\beta \hat{k})$. Instead of the Heisenberg relations one has

$$
\begin{gathered}
\chi_{v}\left(-\alpha_{i} \hat{x}_{i}\right) \chi_{v}\left(-\beta_{j} \hat{k}_{j}\right)=\chi_{v}\left(\alpha_{i} \beta_{j} \delta_{i j}\right) \chi_{v}\left(-\beta_{j} \hat{k}_{j}\right) \chi_{v}\left(-\alpha_{i} \hat{x}_{i}\right), \\
\chi_{v}\left(-\alpha_{i} \hat{x}_{i}\right) \chi_{v}\left(-\alpha_{j} \hat{x}_{j}\right)=\chi_{v}\left(-\alpha_{j} \hat{x}_{j}\right) \chi_{v}\left(-\alpha_{i} \hat{x}_{i}\right), \\
\chi_{v}\left(-\beta_{i} \hat{k}_{i}\right) \chi_{v}\left(-\beta_{j} \hat{k}_{j}\right)=\chi_{v}\left(-\beta_{j} \hat{k}_{j}\right) \chi_{v}\left(-\beta_{i} \hat{k}_{i}\right) .
\end{gathered}
$$

One can introduce the Weyl operator

$$
W_{v}(\alpha \hat{x}, \beta \hat{k})=\chi_{v}\left(\frac{1}{2} \alpha \beta\right) \chi_{v}(-\beta \hat{k}) \chi_{v}(-\alpha \hat{x})
$$

which satisfies relation

$$
W_{v}(\alpha \hat{x}, \beta \hat{k}) W_{v}\left(\alpha^{\prime} \hat{x}, \beta^{\prime} \hat{k}\right)=\chi_{v}\left(\frac{1}{2}\left(\alpha \beta^{\prime}-\alpha^{\prime} \beta\right)\right) W_{v}\left(\left(\alpha+\alpha^{\prime}\right) \hat{x},\left(\beta+\beta^{\prime}\right) \hat{k}\right)
$$

and is a unitary representation of the Heisenberg-Weyl group. Using $W_{v}(\alpha \hat{x}, \beta \hat{k})$ one obtains generalized Weyl formula for quantization

$$
\hat{f}_{v}(\hat{k}, \hat{x})=\int W_{v}(\alpha \hat{x}, \beta \hat{k}) \tilde{f}_{v}(\alpha, \beta) d^{n} \alpha d^{n} \beta
$$

As a basic instrument to treat dynamics of a $p$-adic quantum model is natural to take the kernel $\mathscr{K}_{p}\left(x^{\prime \prime}, t^{\prime \prime} ; x^{\prime}, t^{\prime}\right)$ of the evolution operator $U_{p}\left(t^{\prime \prime}, t^{\prime}\right)$. This kernel obtains by generalization of its real analog, i.e.

$$
\psi_{v}\left(x^{\prime \prime}, t^{\prime \prime}\right)=\int \mathscr{K}_{v}\left(x^{\prime \prime}, t^{\prime \prime} ; x^{\prime}, t^{\prime}\right) \psi_{v}\left(x^{\prime}, t^{\prime}\right) d^{n} x^{\prime},
$$


where $\mathscr{K}_{v}\left(x^{\prime \prime}, t^{\prime \prime} ; x^{\prime}, t^{\prime}\right)$ for quadratic Lagrangians can be defined by path integral

$$
\mathscr{K}_{v}\left(x^{\prime \prime}, t^{\prime \prime} ; x^{\prime}, t^{\prime}\right)=\int_{\left(x^{\prime}, t^{\prime}\right)}^{\left(x^{\prime \prime}, t^{\prime \prime}\right)} \chi_{v}\left(-\int_{t^{\prime}}^{t^{\prime \prime}} L(\dot{q}, q, t) d t\right) \mathscr{D}_{v} q
$$

In the Vladimirov-Volovich formulation [20], $p$-adic quantum mechanics is a triple

$$
\left(L_{2}\left(\mathbb{Q}_{p}\right), W_{p}(z), U_{p}(t)\right)
$$

where $W_{p}(z)$ corresponds to $W_{p}(\alpha \hat{x}, \beta \hat{k})$ defined in (30).

Adelic quantum mechanics [18] is a natural generalization of the above formulation of ordinary and $p$-adic quantum mechanics: $\left(L_{2}(\mathbb{A}), W_{\mathbb{A}}(z), U_{\mathbb{A}}(t)\right)$.

In complex-valued adelic analysis it is worth mentioning an additive character

$$
\chi_{\mathbb{A}}(x)=\chi_{\infty}\left(x_{\infty}\right) \prod_{p} \chi_{p}\left(x_{p}\right)
$$

a multiplicative character

$$
|x|_{\mathbb{A}}^{s}=\left|x_{\infty}\right|_{\infty}^{s} \prod_{p}\left|x_{p}\right|_{p}^{s}, \quad s \in \mathbb{C},
$$

and elementary functions of the form

$$
\varphi_{\mathscr{P}}(x)=\varphi_{\infty}\left(x_{\infty}\right) \prod_{p \in \mathscr{P}} \varphi_{p}\left(x_{p}\right) \prod_{p \notin \mathscr{P}} \Omega\left(\left|x_{p}\right|_{p}\right)
$$

where $\varphi_{\infty}\left(x_{\infty}\right)$ is an infinitely differentiable function on $\mathbb{R}$ and $\left|x_{\infty}\right|_{\infty}^{n} \varphi_{\infty}\left(x_{\infty}\right) \rightarrow 0$ as $\left|x_{\infty}\right|_{\infty} \rightarrow \infty$ for any $n \in\{0,1,2, \cdots\}, \varphi_{p}\left(x_{p}\right)$ are some locally constant functions with compact support, and

$$
\Omega\left(\left|x_{p}\right|_{p}\right)= \begin{cases}1, & \left|x_{p}\right|_{p} \leq 1 \\ 0, & \left|x_{p}\right|_{p}>1\end{cases}
$$

All finite linear combinations of elementary functions (38) make the set $\mathscr{S}(\mathbb{A})$ of the Schwartz-Bruhat adelic functions. The Fourier transform of $\varphi(x) \in \mathscr{S}(\mathbb{A})$, which maps $\mathscr{S}(\mathbb{A})$ onto $\mathscr{S}(\mathbb{A})$, is

$$
\tilde{\varphi}(y)=\int_{\mathbb{A}} \varphi(x) \chi_{\mathbb{A}}(x y) d x,
$$

where $\chi_{\mathbb{A}}(x y)$ is defined by (36) and $d x=d x_{\infty} d x_{2} d x_{3} \cdots$ is the Haar measure on $\mathbb{A}$.

A basis of $L_{2}(\mathbb{A}(\mathscr{P}))$ may be given by the corresponding orthonormal eigenfunctions in a spectral problem of the evolution operator $U_{\mathbb{A}}(t)$, where $t \in \mathbb{A}$. Such eigenfunctions have the form

$$
\psi_{\mathscr{P}}(x, t)=\psi_{\infty}\left(x_{\infty}, t_{\infty}\right) \prod_{p \in \mathscr{P}} \psi_{p}\left(x_{p}, t_{p}\right) \prod_{p \notin \mathscr{P}} \Omega\left(\left|x_{p}\right|_{p}\right)
$$


where $\psi_{\infty} \in L_{2}(\mathbb{R})$ and $\psi_{p} \in L_{2}\left(\mathbb{Q}_{p}\right)$ are eigenfunctions in ordinary and $p$-adic cases, respectively. $\Omega\left(\left|x_{p}\right|_{p}\right)$ is an element of $L_{2}\left(\mathbb{Q}_{p}\right)$, defined by (39), which is invariant under transformation of an evolution operator $U_{p}\left(t_{p}\right)$ and provides convergence of the infinite product (41). Elements of $L_{2}(\mathbb{A})$ may be regarded as superpositions $\psi(x)=\sum_{\mathscr{P}} C(\mathscr{P}) \psi_{\mathscr{P}}(x)$, where $\psi_{\mathscr{P}}(x) \in L_{2}(\mathbb{A}(\mathscr{P}))(41)$ and $\sum_{\mathscr{P}}|C(\mathscr{P})|_{\infty}^{2}=1$.

Theory of $p$-adic generalized functions is presented in [6] and a theory of generalized functions on adelic spaces is in progress [25].

Adelic evolution operator $U_{\mathbb{A}}(t)$ is defined by

$$
\begin{aligned}
U_{\mathbb{A}}\left(t^{\prime \prime}\right) \psi\left(x^{\prime \prime}\right) & =\int_{\mathbb{A}} \mathscr{K}_{\mathbb{A}}\left(x^{\prime \prime}, t^{\prime \prime} ; x^{\prime}, t^{\prime}\right) \psi\left(x^{\prime}, t^{\prime}\right) d x^{\prime} \\
& =\prod_{v} \int_{\mathbb{Q}_{v}} \mathscr{K}_{v}\left(x_{v}^{\prime \prime}, t_{v}^{\prime \prime} ; x_{v}^{\prime}, t_{v}^{\prime}\right) \psi_{v}\left(x_{v}^{\prime}, t_{v}^{\prime}\right) d x_{v}^{\prime} .
\end{aligned}
$$

The eigenvalue problem for $U_{\mathbb{A}}(t)$ reads

$$
U_{\mathbb{A}}(t) \psi_{\mathscr{P}}(x)=\chi_{\mathbb{A}}\left(E_{\alpha} t\right) \psi_{\mathscr{P}}(x)
$$

where $\psi_{\mathscr{P}}(x)$ are adelic eigenfunctions $(41)$, and $E_{\alpha}=\left(E_{\infty}, E_{2}, \ldots, E_{p}, \ldots\right)$ is the corresponding adelic energy.

Adelic quantum mechanics takes into account ordinary as well as $p$-adic quantum effects and may be regarded as a starting point for construction of a more complete quantum cosmology [18], quantum field theory [26] and string/M-theory [27, 28]. In the limit of large distances adelic quantum mechanics effectively becomes the ordinary one [29].

Evaluation of $v$-adic kernel $\mathscr{K}_{v}\left(x^{\prime \prime}, t^{\prime \prime} ; x^{\prime}, t^{\prime}\right)$ of the unitary evolution operator for onedimensional systems with quadratic Lagrangians has the form [30, 31, 32]

$$
\begin{aligned}
\mathscr{K}_{v}\left(x^{\prime \prime}, t^{\prime \prime} ; x^{\prime}, t^{\prime}\right)= & \lambda_{v}\left(-\frac{1}{2 h} \frac{\partial^{2}}{\partial x^{\prime \prime} \partial x^{\prime}} \bar{S}\left(x^{\prime \prime}, t^{\prime \prime} ; x^{\prime}, t^{\prime}\right)\right) \\
& \times\left|\frac{1}{h} \frac{\partial^{2}}{\partial x^{\prime \prime} \partial x^{\prime}} \bar{S}\left(x^{\prime \prime}, t^{\prime \prime} ; x^{\prime}, t^{\prime}\right)\right|_{v}^{\frac{1}{2}} \chi_{v}\left(-\frac{1}{h} \bar{S}\left(x^{\prime \prime}, t^{\prime \prime} ; x^{\prime}, t^{\prime}\right)\right),
\end{aligned}
$$

where $\lambda_{v}$-functions are presented in [6].

\section{2. $p$-Adic and adelic wave functions of the universe}

The universe should be a quantum system, especially at the very beginning of its evolution. The main task of quantum cosmology is to provide formalism to specify quantum states and describe quantum dynamics of the universe as a whole. The quantum state of the universe at the beginning is of central importance, since it determines initial condition for its later behavior. Considering the universe as a quantum-mechanical system it has a quantum state which is encoded in the corresponding wave function. 
This wave function is complex-valued and depends on some real quantities in standard approach. To include $p$-adic effects one has to reconsider its formulation. We maintain here the standard point of view that the wave function takes complex values, but we treat its arguments (space-time coordinates, gravitational and matter fields) to be not only real but also $p$-adic and adelic.

There is not $p$-adic generalization of the Wheeler - De Witt equation for cosmological models. Instead of differential approach, Feynman's path integral method in the HartleHawking approach [33] was exploited [18] and minisuperspace cosmological models are also investigated by means of adelic quantum mechanics [16].

$p$-Adic and adelic minisuperspace quantum cosmology is an application of $p$-adic and adelic quantum mechanics to the cosmological models, respectively. In the path integral approach to standard quantum cosmology, the starting point is Feynman's path integral method. The amplitude to go from one state with intrinsic metric $h_{i j}^{\prime}$ and matter configuration $\phi^{\prime}$ on an initial hypersurface $\Sigma^{\prime}$ to another state with metric $h_{i j}^{\prime \prime}$ and matter configuration $\phi^{\prime \prime}$ on a final hypersurface $\Sigma^{\prime \prime}$ is given by the path integral

$$
\mathscr{K}_{\infty}\left(h_{i j}^{\prime \prime}, \phi^{\prime \prime}, \Sigma^{\prime \prime} ; h_{i j}^{\prime}, \phi^{\prime}, \Sigma^{\prime}\right)=\int \chi_{\infty}\left(-S_{\infty}\left[g_{\mu \nu}, \Phi\right]\right) \mathscr{D}_{\infty} g_{\mu \nu} \mathscr{D}_{\infty} \Phi
$$

over all four-geometries $g_{\mu \nu}$ and matter configurations $\Phi$, which interpolate between the initial and final configurations. In (45) $S_{\infty}\left[g_{\mu \nu}, \Phi\right]$ is an Einstein-Hilbert action for the gravitational and matter fields. To perform $p$-adic and adelic generalization we make first $p$-adic counterpart of the action using form-invariance under change of real to the $p$-adic number fields. Then we generalize (45) and introduce $p$-adic complex-valued cosmological amplitude

$$
\mathscr{K}_{p}\left(h_{i j}^{\prime \prime}, \phi^{\prime \prime}, \Sigma^{\prime \prime} ; h_{i j}^{\prime}, \phi^{\prime}, \Sigma^{\prime}\right)=\int \chi_{p}\left(-S_{p}\left[g_{\mu \nu}, \Phi\right]\right) \mathscr{D}_{p} g_{\mu \nu} \mathscr{D}_{p} \Phi
$$

The standard minisuperspace ground-state wave function in the Hartle-Hawking (noboundary) proposal [33] is defined by functional integration in the Euclidean version of

$$
\psi_{\infty}\left[h_{i j}\right]=\int \chi_{\infty}\left(-S_{\infty}\left[g_{\mu \nu}, \Phi\right]\right) \mathscr{D}_{\infty} g_{\mu \nu} \mathscr{D}_{\infty} \Phi
$$

over all compact four-geometries $g_{\mu \nu}$ which induce $h_{i j}$ at the compact three-manifold. This three-manifold is the only boundary of the all four-manifolds. Extending HartleHawking proposal to the $p$-adic minisuperspace [15], an adelic Hartle-Hawking wave function is the infinite product

$$
\psi_{\mathbb{A}}(q)=\prod_{v} \int \chi_{v}\left(-S_{v}\left[g_{\mu v}, \Phi\right]\right) \mathscr{D}_{\nu} g_{\mu \nu} \mathscr{D}_{v} \Phi
$$

where path integration must be performed over both, Archimedean and nonArchimedean geometries. If an evaluation of the corresponding functional integrals for a minisuperspace model yields $\psi\left(q_{\alpha}\right)$ in the form (41), then such cosmological model is a Hartle-Hawking adelic one. 
Before to proceed in the above way it is worth mentioning another approach [15] which was the first one in $p$-adic quantum cosmology. The essence of this approach consists in the following $p$-adic proposal for the Hartle-Hawking type of the wave function:

$$
\psi_{\infty}(q)=\sum_{a . m .} \prod_{p} \int \chi_{p}\left(-S_{p}\left[g_{\mu v}, \Phi\right]\right) \mathscr{D}_{p} g_{\mu \nu} \mathscr{D}_{p} \Phi
$$

where summation is over algebraic manifolds. This proposal was illustrated on the above de Sitter model with the Euclidean version of $d s^{2}$ in (14).

It is shown [18] that the de Sitter minisuperspace model in $D=4$ space-time dimensions is the Hartle-Hawking adelic one. Namely, according to the Hartle-Hawking proposal one has

$$
\psi_{v}(q)=\int \mathscr{K}_{v}(q, T ; 0,0) d T, \quad v=\infty, 2,3, \cdots, p, \cdots
$$

where

$$
\mathscr{K}_{v}\left(q^{\prime \prime}, T ; q^{\prime}, 0\right)=\lambda_{v}(-8 T)|4 T|_{v}^{-\frac{1}{2}} \chi_{v}\left[-\frac{\lambda^{2} T^{3}}{24}+(\lambda q-2) \frac{T}{4}+\frac{q^{2}}{8 T}\right]
$$

is the kernel of the $v$-adic evolution operator. The functions $\lambda_{v}(a)$ have the properties [6]

$$
\left|\lambda_{v}(a)\right|_{v}=1, \lambda_{v}\left(b^{2} a\right)=\lambda_{v}(a), \lambda_{v}(a) \lambda_{v}(b)=\lambda_{v}(a+b) \lambda_{v}(a b(a+b)) .
$$

Employing the $p$-adic Gauss integral [6]

$$
\int_{\mathbb{Q}_{p}} \chi_{p}\left(\alpha x^{2}+\beta x\right) d x=\lambda_{p}(\alpha)|2 \alpha|_{p}^{-\frac{1}{2}} \chi_{p}\left(-\frac{\beta^{2}}{4 \alpha}\right), \quad \alpha \neq 0,
$$

one can rewrite $p$-adic version of (50) in the form

$$
\psi_{p}(q)=\int_{\mathbb{Q}_{p}} d x \chi_{p}(q x) \int D T \chi_{p}\left[-\frac{\lambda^{2} T^{3}}{24}+\left(\frac{\lambda q}{4}-\frac{1}{2}-2 x^{2}\right) T\right] .
$$

Taking the region of integration to be $|T|_{p} \leq 1$ one obtains

$$
\psi_{p}(q)=\int_{\mathbb{Q}_{p}} d x \chi_{p}(q x) \Omega\left(\left|\frac{\lambda q}{4}-\frac{1}{2}-2 x^{2}\right|_{p}\right), \quad\left|\frac{\lambda^{2}}{24}\right|_{p} \leq 1 .
$$

An evaluation of the integral (55) yields

$$
\psi_{p}(q)=\exp \left(i \pi \delta_{|q|_{2}}^{1} \delta_{p}^{2}\right) \Omega\left(|q|_{p}\right), \quad\left|\frac{\lambda^{2}}{24}\right|_{p} \leq 1
$$

where $\delta_{a}^{b}$ is the Kronecker symbol. $\psi_{\infty}\left(q_{\infty}\right)$ was explored in [17] and the result depends on the contour of integration and has an exact solution

$$
\psi_{\infty}\left(q_{\infty}\right)=\exp \left(\frac{1}{3 \lambda}\right) \operatorname{Ai}\left(\frac{1-\lambda q_{\infty}}{(2 \lambda)^{\frac{2}{3}}}\right)
$$


where $\operatorname{Ai}(x)$ is the Airy function. Finally we obtain an adelic wave function for the de Sitter cosmological model in the form

$$
\psi_{\mathbb{A}}(q)=\psi_{\infty}\left(q_{\infty}\right) \prod_{p} \exp \left(i \pi \delta_{|q|_{2}}^{1} \delta_{p}^{2}\right) \Omega\left(\left|q_{p}\right|_{p}\right), \quad\left|\frac{\lambda^{2}}{24}\right|_{p} \leq 1 .
$$

It is shown in $[19,16]$ that $p$-adic and adelic generalization of the minisuperspace cosmological models can be successfully performed in the framework of $p$-adic and adelic quantum mechanics $[21,22]$ without use of the Hartle-Hawking approach. The following cosmological models are investigated [16]: the de Sitter model, model with a homogeneous scalar field, anisotropic Bianchi model with three scale factors and some two-dimensional minisuperspace models. The necessary condition that a system can be regarded as the adelic one is the existence of $p$-adic ground state $\Omega\left(\left|q_{\alpha}\right|_{p}\right)$ $(\alpha=1,2, \cdots, n)$ in the way

$$
\int_{\left|q_{\alpha}^{\prime}\right|_{p} \leq 1} \mathscr{K}_{p}\left(q_{\alpha}^{\prime \prime}, T ; q_{\alpha}^{\prime}, 0\right) d q_{\alpha}^{\prime}=\Omega\left(\left|q_{\alpha}^{\prime \prime}\right|_{p}\right)
$$

for all $p$ but a finite set $\mathscr{P}$. For the case of de Sitter model one obtains

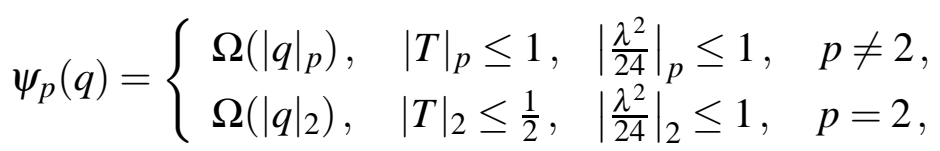

what is in a good agreement with the result (58) obtained by the Hartle-Hawking proposal. Some other forms of the $p$-adic wave functions are also found [16], e.g. $\psi_{p}(q)=\Omega\left(p^{\mu}|q|_{p}\right)$ and $\psi_{p}(q)=\delta\left(|q|_{p}-p^{\mu}\right)$ where $\mu \in \mathbb{Z}$.

Let us now consider the interpretation of an adelic wave function. Note that there is a general problem of the usual interpretation of the wave function of the universe. Here we are going to discuss only adelic aspects. It is natural to investigate adelic wave function on the rational values of its arguments. Without loss of generality we can use the above de Sitter cosmological model. For the adelic ground state (58) the density of probability in rational points is

$$
\left|\psi_{\mathbb{A}}(q)\right|_{\infty}^{2}=\left|\psi_{\infty}(q)\right|_{\infty}^{2} \prod_{p} \Omega\left(|q|_{p}\right)= \begin{cases}\left|\psi_{\infty}(q)\right|_{\infty}^{2}, & q \in \mathbb{Z}, \\ 0, & q \in \mathbb{Q} \backslash \mathbb{Z} .\end{cases}
$$

When (the density of) probability is equal (or close) to unity or zero it can be regarded as a certain event. From (61) it follows that $q$-space is discrete, because the density probability is nonzero only at integers. Note that here spacing is related to the Planck length but in some other adelic quantum models it will correspond to the characteristic length of the system. If we integrate (58) over $\left|q_{p}\right|_{p} \leq 1$ for every $p$ then we obtain ordinary wave function. In the case of adelic wave function of the form (41) with $\mathscr{P} \neq \emptyset$, $q$-space has not a sharp discreteness but fuzzyness.

As a result of $p$-adic effects, adelic quantum systems contain discreteness of the space with spacing equal to the own characteristic length. This kind of discreteness was 
obtained for the first time in the context of the Hartle-Hawking adelic de Sitter quantum model [18]. At the distances much larger than the characteristic length instead of an adelic wave function it is enough to use only the ordinary one over real space.

\section{REAL AND $p$-ADIC WORLDS. ADELIC UNIVERSE}

\subsection{On Adelic Models}

Let us introduce two distinct types of adelic models: $(i)$ principal adelic models and (ii) non-principal adelic models.

In principal adelic models all parameters and constants, which characterize physical system, are principal ideles, i.e. they are nonzero rational numbers which are the same in real and all $p$-adic counterparts. Adelic product formulas can exist in these models, which connect real and $p$-adic aspects of the same quantity. A simple and illustrative such formula is

$$
|x|_{\infty}^{c} \prod_{p}|x|_{p}^{c}=1, \quad c \in \mathbb{C}, \quad x \in \mathbb{Q} \backslash\{0\},
$$

which is adelic multiplicative character on principal ideles. Another simple example is adelic additive character on principal adeles, i.e.

$$
\chi_{\infty}(x) \prod_{p} \chi_{p}(x)=1, \quad x \in \mathbb{Q} .
$$

Employing $|x|_{v}^{\alpha-1}$ and $\chi_{v}(x)$ in some integrals one can obtain new product formulas. For instance (see [34] and references therein)

$$
\begin{gathered}
\Gamma_{\infty}(\alpha) \prod_{p} \Gamma_{p}(\alpha)=1, \quad \Gamma_{v}(\alpha)=\int_{\mathbb{Q}_{v}}|x|_{v}^{\alpha-1} \chi_{v}(x) d_{v} x, \quad \alpha \neq 0,1, \\
B_{\infty}(a, b) \prod_{p} B_{p}(a, b)=1, \quad B_{v}(a, b)=\int_{\mathbb{Q}_{v}}|x|_{v}^{a-1}|1-x|_{v}^{b-1} d_{v} x, \quad a+b+c=1 .
\end{gathered}
$$

While the formulas (62) and (63) are valid only for rational numbers, (64) and (65) have place for real and complex numbers. The product formula (65) connects real and $p$-adic amplitudes for scattering of two open string tachyons [8]. As a result of (65), complicated real amplitude can be expressed as product of inverse $p$-adic counterparts which are elementary functions. There are also some product formulas which include all $p$-adic counterparts but not the real one. Such an example is

$$
\prod_{p} \Omega\left(|x|_{p}\right)= \begin{cases}1, & x \in \mathbb{Z}, \\ 0, & x \in \mathbb{Q} \backslash \mathbb{Z} .\end{cases}
$$

We used (66) in (61). 
Adelic quantum mechanics [21, 22] and its generalizations to quantum field theory [26] and string theory [27] belong to the principal adelic models.

In non-principal adelic models parameters and constants are adeles but not principal ones, i.e. they are not rational numbers the same for real and $p$-adic counterparts. A non-principal adelic constant (or parameter) is an adele

$$
c_{\mathbb{A}}=\left(c_{\infty}, c_{2}, c_{3}, \cdots, c_{p}, \cdots\right)
$$

where at least two of components $c_{v}$ are not the same rational number. There are infinitely many possibilities to fix all $c_{v}$ in (67). One of them is the case when $c_{p}=p$ for all primes $p$. Another interesting case is when all but a finite number of $c_{v}$ are equal zero. In particular, all but one of $c_{v}$ can be zero. If only $c_{\infty} \neq 0$, adelic model is reduced to the real one. Analogously if only $c_{p} \neq 0$ for a fixed $p$, we have a p-adic model. In the sequel we will pay some attention to the case when $c_{\infty} \neq 0$ and $c_{p} \neq 0$ for a finite set $\mathscr{P}$ of primes $p$.

\section{2. $p$-Adic Matter, Dark Energy and Dark Matter}

Applying classical Einstein equations (8) and the FRW metric (13) (with $N=1, c=1$ ) to the universe, one obtains

$$
\frac{\ddot{a}}{a}=-\frac{4 \pi G}{3} \sum_{i}\left(\rho_{i}+3 p_{i}\right)+\frac{\Lambda}{3}, \quad\left(\frac{\dot{a}}{a}\right)^{2}+\frac{k}{a^{2}}=\frac{8 \pi G}{3} \sum_{i} \rho_{i}+\frac{\Lambda}{3},
$$

where $\rho_{i}$ and $p_{i}$ are $i$-th component of energy and pressure density, respectively, and $a$ is cosmological scale factor. The equation of state is $p_{i}=w_{i} \rho_{i}$. Since $\Lambda$ can be regarded as an energy density we can rewrite equations (68) in the following form

$$
\begin{aligned}
& \frac{\ddot{a}}{a}=-\frac{4 \pi G}{3} \sum_{i}\left(\rho_{i}+3 p_{i}\right), \\
& \left(\frac{\dot{a}}{a}\right)^{2}+\frac{k}{a^{2}}=\frac{8 \pi G}{3} \sum_{i} \rho_{i} .
\end{aligned}
$$

Recent observations (see reviews [2, 3]) show that

$$
\Omega=\sum_{i} \frac{\rho_{i}}{\rho_{c r}}=\sum_{i} \Omega_{i} \approx 1, \quad \Omega_{B} \approx 0.04, \quad \Omega_{D M} \approx 0.26, \quad \Omega_{D E} \approx 0.70
$$

where $\Omega_{B}, \Omega_{D M}$ and $\Omega_{D E}$ are related to baryonic matter, dark matter and dark energy, respectively, and $\rho_{c r}=\frac{3}{8 \pi G} H_{0}^{2}$ is critical energy density required for the universe to be spatially flat. $H_{0}=\left(\frac{\dot{a}}{a}\right)_{0}$ is the rate of expansion at present time. While baryonic matter is partially luminous, the nature of dark matter and dark energy is presently unknown.

Dark matter was introduced (already in 1930's) to explain dynamics of galaxies in clusters as well as dynamics inside individual galaxies. It is nonbaryonic and clustered 
(attractive) matter. Many nonbaryonic particles (in particular, neutralino and axion) have been considered as candidates for dark matter.

Dark energy was introduced as an unclustered (repulsive) matter with negative pressure and responsible for accelerated expansion of the universe. Cosmic acceleration was discovered recently $[35,36]$ in observations of cosmologically distant Type Ia Supernovae (SNeIa) and independently verified by Cosmic Microwave Background and large scale structure investigations. Namely, to have an acceleration ( $\ddot{a}>0)$, the RHS of eq. (69) has to be positive, i.e. $\sum_{i}\left(\rho_{i}+3 p_{i}\right)<0$. Since $\rho_{B}+3 p_{B}>0$ and $\rho_{D M}+3 p_{D M}>0$ it follows that $\rho_{D E}+3 p_{D E}<0$. Positivity of $\rho_{D E}$ yields $p_{D E}<-\frac{\rho_{D E}}{3}<0$, i.e. $w<-1 / 3$. Current observational value of parameter $w$ (see [2] and references therein) is in the range $-1.61<w<-0.78$.

There are many candidate models for the dark energy. The simplest model is the cosmological constant with $w=-1 \quad(p=-\rho=$ const . $)$. Some other dynamical scalar field models are [2]: quintessence, braneworld, Chaplygin gas and Phantom dark energy (with $w<-1$ ). In spite of many interesting results, neither these nor other so far proposed models offer satisfactory answers to all questions around the dark energy.

I propose here $p$-adic origin of the dark energy and dark matter. In other words, dark energy and dark matter are two forms of the $p$-adic matter. If we take string theory seriously then there exist not only real strings but also $p$-adic strings. Real and $p$-adic matter consist of real and $p$-adic strings, respectively. Even without string theory, using $p$-adic quantum-mechanical models one can argue possible existence of $p$-adic matter.

An instructive model of $p$-adic matter is nonlocal and nonlinear effective scalar field theory $[9,10]$ which describes dynamics of $p$-adic tachyonic matter. The corresponding action is given by

$$
S=\int d^{d} x \mathscr{L}_{p}, \quad \mathscr{L}_{p}=\frac{1}{g_{p}^{2}}\left[-\frac{1}{2} \phi p^{-\frac{1}{2} \square} \phi+\frac{1}{p+1} \phi^{p+1}\right],
$$

where

$$
\frac{1}{g_{p}^{2}}=\frac{1}{g^{2}} \frac{p^{2}}{p-1}, \quad \square=-\frac{\partial^{2}}{\partial t^{2}}+\frac{\partial^{2}}{\partial x_{1}^{2}}+\cdots+\frac{\partial^{2}}{\partial x_{d-1}^{2}}
$$

and the equation of motion is

$$
p^{-\frac{1}{2} \square} \phi=\phi^{p}
$$

This $p$-adic string theory was employed in the context of tachyon condensation [11] and for a recent review see [37]. Some mathematical aspects of time dependent solutions of (74) were analyzed in [38] (see also references therein). It is worth mentioning that this $p$-adic tachyon theory resembles dynamics of nonlocal real tachyon in string field theory, which is also under consideration as a candidate for cosmological dark energy [39]. It would be of great importance to find $p$-adic valued counterpart of Lagrangian (72) which reproduces the same complex-valued tachyon amplitudes at the tree level, as well as to establish a map between them. 
According to this proposal baryonic matter is real one, while dark energy and dark matter have $p$-adic origin. Work on a concrete $p$-adic model of dark energy and dark matter is in progress.

\subsection{Adelic Universe with Real and $p$-Adic Worlds}

An example of a non-principal adelic physical system could be the universe as a whole. Namely, it seems natural to regard our universe as adelic one consisting of real and $p$-adic worlds which interact only gravitationally. Each of these worlds is made of its own matter and their main characteristics can be described by relevant real or $p$-adic numbers.

Even if we suppose that at the very beginning the universe was as a principal adelic system it could evolve to the non-principal one. Namely, according to string landscape (see recent discussion in [40]), effective potential in string theory has many local maxima and minima which determine values of various parameters. During evolution they can lead to different values of parameters in real and $p$-adic sectors and definitely to transformation of principal adelic strings to real and $p$-adic ones, which may be regarded as non-principal adelic strings.

\section{CONCLUDING REMARKS}

In an introductory way we have reviewed basic properties of $p$-adic and adelic cosmology. Proposal on $p$-adic origin of dark energy and dark matter, as well as on $p$-adic worlds of the universe, has been put forward.

It is worth mentioning some coincidences between $p$-adics and dimensionality of the space-time. A nonsingular form of an odd degree $r$ [41]

$$
\sum_{i=1}^{n} a_{i} x_{i}^{r}, \quad a_{i} \in \mathbb{Q}, \quad r=2 k+1, \quad k \in \mathbb{N} \cup\{0\}
$$

represents all $p$-adic numbers in a nontrivial way in $\mathbb{Q}_{p}$ if $n \geq D=r^{2}+1$. Also the above form (75) nontrivially represents all rational numbers in $\mathbb{Q}$ when $n \geq D=r^{2}+1$. It follows that the lowest dimension $D$ for $r=3$ is $D=10$ and for $r=5, D=26$. It was noted [42] that these dimensions $D$ coincide with critical dimensions of space-time in string theory, i.e. $D=10$ for superstrings and $D=26$ for bosonic strings.

Another intriguing coincidence is related to quadratic algebraic extensions (of $\mathbb{R}$ and $\left.\mathbb{Q}_{p}\right)$ and dimensionality of M-theory. Recall [6] that in real, $p$-adic $(p \neq 2)$ and 2-adic cases we have one, three and seven quadratic extensions, respectively. Their sum gives $1+3+7=11$, what is just number of space-time dimensions in M-theory. 
$p$-Adic pseudoconstants [43] are $p$-adic valued functions $c_{p}(t)$ which have derivatives vanishing, i.e. $\dot{c}_{p}(t)=0$ for $t \in \mathbb{Z}_{p}$. They give new cosmological possibilities comparing with real analysis and could be related [15] to stochastic inflation.

\section{ACKNOWLEDGMENTS}

The work on this article has been supported by the Ministry of Science and Environmental Protection of the Republic of Serbia under projects No 1426 and No 144032. I would like to thank I.Ya. Aref'eva, V.S. Vladimirov and I.V. Volovich for inspirative and useful discussions I have had with them in various periods of work on some problems contained in this paper.

\section{REFERENCES}

1. D. Scott, The Standard Cosmological Model, astro-ph/ 0510731.

2. V. Sahni, Dark Matter and Dark Energy, ast ro-ph/ 0403324.

3. T. Padmanabhan, Darker Side of the Universe, a st ro-ph/0510492.

4. W.H. Schikhof, Ultrametric Calculus: An Introduction to $p$-Adic Analysis, Cambridge Univ. Press, Cambridge, 1984.

5. I.M. Gel'fand, M.I. Graev and I.I. Pyatetski-Shapiro, Representation Theory and Automorphic Functions, Saunders, Philadelphia, 1969.

6. V.S. Vladimirov, I.V. Volovich and E.I. Zelenov, p-Adic Analysis and Mathematical Physics, World Scientific, Singapore, 1994.

7. I.V. Volovich, Class. Quantum Grav. 4, L83-L87 (1987).

8. P.G.O. Freund and E. Witten, Phys. Lett. B 199, 191-194 (1987).

9. L. Brekke, P.G.O. Freund, M. Olson and E. Witten, Nucl. Phys. B 302, 365 (1988).

10. P.H. Frampton and Y. Okada, Phys. Rev. D 37, 3077-3079 (1988).

11. D. Ghoshal and A. Sen, Nucl. Phys. B 584, 300-312 (2000).

12. L. Brekke and P.G.O. Freund, Phys.Rept. 233, 1-66 (1993).

13. Selected Topics of $p$-Adic Mathematical Physics, Proc. of the 1st Int. Conf. on $p$-Adic Math. Physics (Moscow, 2003), Proc V.A. Steklov Inst. Math. 245, Nauka, Moscow, 2004.

14. $p$-Adic Mathematical Physics 2005, Proc. of the 2nd Int. Conf. on $p$-Adic Math. Physics (Belgrade, 2005), AIP Conference Proceedings, American Institute of Physics, New York, 2006.

15. I.Ya. Aref'eva, B. Dragovich, P.H. Frampton and I.V. Volovich, Int. J. Mod. Phys. A 6, 4341-4358 (1991).

16. G.S. Djordjević, B. Dragovich, L.D. Nešić and I.V. Volovich, Int. J. Mod. Phys. A 17, 1413-1433 (2002), gr-qc/0105050.

17. J.J. Halliwell and J. Louko, Phys. Rev. D 39, 2206 (1989).

18. B. Dragovich, Adelic Wave Function of the Universe, in Proc. the Third A. Friedmann Int. Seminar on Grav. and Cosmology, St. Petersburg, pp. 311-321 (1995); Adelic Wave Function of the de Sitter Universe, in Proc. 7th Lomonosov Conf. on Elem. Particle Physics, Moscow, pp. 137-140 (1995); Adelic Aspects of Quantum Cosmology, in Proc. 8th Lomonosov Conf. on Elem. Particle Physics, Moscow, pp. 116-122 (1997).

19. B. Dragovich and Lj. Nešić, Grav. Cosm. 5, 222 (1999), gr-qc/ 0005103.

20. V.S. Vladimirov and I.V. Volovich, Commun. Math. Phys. 123, 659 (1989). 
21. B. Dragovich, Teor. Mat. Fizika 101, 349-359 (1994), hep-th/ 0402193.

22. B. Dragovich, Int. J. Mod. Phys. A 10, 2349-2365 (1995), hep-th/ 0404160 .

23. B. Dragovich, $p$-Adic and Adelic Quantum Mechanics, Proc. V. A. Steklov Inst. Math. 245, 72-85, (2004), hep-th/ 0312046.

24. A. Yu. Khrennikov, p-Adic Valued Distributions in Mathematical Physics, Kluwer, Dordrecht, 1994.

25. B. Dragovich, Integral Transforms and Spec. Funct. 6, 197-203 (1998), math-ph/ 0404076.

26. B. Dragovich, Nucl. Phys. B (Proc. Suppl.) 102/103, 150-155 (2001).

27. B. Dragovich, $p$-Adic and Adelic Strings, in Proc. Int. Conf. Dedicated to the Memory of Prof. E. Fradkin: Quantization, Gauge Theory and Strings, Moscow, pp. 108-114 (2001).

28. B. Dragovich and A. Khrennikov, $p$-Adic and Adelic Superanalysis, hep-th/ 0512318 .

29. G.S. Djordjević, B. Dragovich and Lj. Nešić, Mod. Phys. Lett. A 14, 317-325 (1999), hep-th/ 0005216.

30. G.S. Djordjević and B. Dragovich, On p-Adic Functional Integration, in Proc. II Mathematical Conf. in Priština, ed. Lj. D. Kočinac, University of Priština, 1997, pp. 221 - 227; math-ph/ 0005025.

31. G.S. Djordjević and B. Dragovich, Mod. Phys. Lett. A 12, 1455-1463 (1997), math-ph/ 0005026.

32. G.S. Djordjević, B. Dragovich and L. Nešić, Inf. Dim. Analys. Quan. Probab. and Rel. Topics 6, 176-195 (2003), hep-th/ 0105030.

33. J. Hartle and S. Hawking, Phys. Rev. 28, 2960 (1983).

34. V.S. Vladimirov, Adelic Formulas for Four-Particle String and Superstring Tree Amplitudes in OneClass Quadratic Fields, in Proc. of the 1st Int. Conf. on p-Adic Math. Physics (Moscow, 2003), Proc V.A. Steklov Inst. Math. 245, Nauka, Moscow, pp. 9-28 (2004).

35. S.J. Perlmutter et al., Astroph. J. 517, 5651999.

36. A. Riess et al., Astron. J. 116, 10091998.

37. P.G.O. Freund, $p$-Adic Strings and Their Applications, in these Proceedings, hep-th/ 0510192.

38. V.S. Vladimirov and Ya.I. Volovich, On the Nonlinear Dynamical Equation in the $p$-Adic String Theory, math-ph/0306018.

39. I.Ya. Aref'eva, Nonlocal String Tachyon as a Model for Cosmological Dark Energy, in these Proceedings.

40. S. Weinberg, Living in the Multiverse, hep-th/0511037.

41. Z.I. Borevich and I.R. Shafarevich, Number Theory, Academic Press, 1966.

42. B. Dragovich, Mod. Phys. Lett. A 6, 2301-2307 (1991).

43. K. Mahler, Introduction to $p$-Adic Numbers and Their Functions, Cambridge Univ. Press, Cambridge, 1973. 